gativi. La PFGE dei CTX-M produttori ha rivelato eterogeneità clonale con quattro linee maggiori (A-D) ed un cluster epidemico in ogni struttura. Plasmidi coniugativi di 50 e $55 \mathrm{~Kb}$ sono stati evidenziati in ceppi di pulsotipo D e B rispettivamente.

Conclusioni: Le strutture geriatriche rappresentano un reservoir di E.coli produttori di ESßL, in particolare di tipo CTXM. La diffusione di geni bla cтх-м in E. coli è sia plasmide- che clone- mediata e, pertanto, destinata ad aumentare. Sono auspicabili misure di controllo efficaci, compreso il monitoraggio dei pazienti portatori di cateteri vescicali colonizzati/infetti da Enterobatteri produttori di ESßL.

\title{
172
}

\section{DIFFUSIONE EPIDEMICA DI E. COLI PRODUTTORI DI ESBL DITIPO CTX-M IN OSPEDALI GERIATRICI.}

'Nucleo E., 'Migliavacca R., ${ }^{2}$ Balzaretti M., ${ }^{3}$ Spalla M., 'Quatela M., ${ }^{3}$ Terulla C., 'Martino F., 'Pagani L..

'Dip. S.M.E.C., sezione di Microbiologia, via Brambilla 74, 27l 00 Pavia,

2Lab. di Microbiologia ASP Piero Redaelli, via B. d'Alviano, 74, 20146, Milano,

3Lab. di Microbiologia IRCCS S. Matteo,

v.le C.Golgi, 19, 27100 Pavia, Italia.

Introduzione: Gli Enterobatteri produttori di ß-lattamasi a spettro esteso (ESßL) tipicamente causano infezioni nosocomiali. Gli enzimi di tipo CTX-M stanno assumendo un ruolo rilevante nel mondo; in Italia, sono stati isolati da pazienti ospedalizzati ed ambulatoriali. Scopo dello studio è determinare prevalenza e diffusione di ESßL di tipo CTX-M in E. coli isolati in tre Ospedali Geriatrici.

Metodi: Nel periodo Marzo 2003- Maggio 2004, presso il Laboratorio di Microbiologia ASP P.Redaelli sono stati raccolti, da urine di pazienti con catetere vescicale, 77 isolati consecutivi, non replicati, di E. coli ESßL produttori (test CLSI). Le B-lattamasi sono state studiate con IEF, attività enzimatica, metodi molecolari per evidenziare i geni bla тем, blasнv, bla стх-м. Sono state effettuate coniugazione, estrazione alcalina dei plasmidi; genotipizzazione mediante PFGE.

Risultati: $61 / 77$ isolati di $E$. coli $\mathrm{ES} \beta L$ positivi esibivano più alti livelli di resistenza al cefotaxime che al ceftazidime. 52/61 isolati presentavano bande $\beta$-lattamasiche multiple; di questi, 9/61 ceppi esprimevano ß-lattamasi di tipo CTX-M, 29/61 producevano enzimi di tipo CTX-M e TEM-1, 7/61 ceppi esprimevano enzimi di tipo CTX-M ed SHV; in 16 casi erano rilevabili geni bla $a_{\text {стх-м }}$, bla тем $_{\text {т }}$ blasнv.

Gli ESßL produttori erano multiresistenti, ma sensibili a piperacillina-tazobactam. La produzione di ß-lattamasi di tipo CTX-M era mediata prevalentemente da plasmidi coniu- 